\title{
Positive Controllability Test of Discrete-Time Linear Systems
}

\author{
Hiroshi Yoshida*, Tetsuro Tanaka* and Kazutomo YunOKuchi*
}

\begin{abstract}
The controllability with positive input constraints is very important and interesting in dealing with many practical control applications such as a pendulum system, economic system, etc. Although the necessary and sufficient condition for the controllability of discrete-time linear systems with positive controls is given earlier, it is not easy to determine whether a given multiple input system is positive controllable or not based on the former result.

This paper presents simple criteria for multiple input discrete-time linear systems to become positive controllable based on the Jordan canonical form and elimination method of Gauss. It is pointed out that the number of steps necessary to discriminate the positive controllability is finite. The results presented here clarify the structure of positive controllable systems and are useful in discussing fundamental control problems.
\end{abstract}

Key Words : controllability with input constraints, positive control, discrete-time linear systems, multiple input systems, Jordan canonical form

\section{Introduction}

Since the concept of the controllability of systems was first introduced by Kalman ${ }^{1)}$ the study of controllability has been one of the most fundamental and essential subjects in system control theory. Wing and Desoer ${ }^{2)}$ gave the necessary and sufficient conditions for multiple-input linear time-invariant control systems to be controllable under input constraints due to saturation which are significant from the viewpoint of industrial applications. Kodama ${ }^{3)}$ generalized the results of Wing and Desoer to the case where the state transition matrix has null eigenvalues. On the other hand the controllability with positive input constraints is very important and interesting in dealing with many practical control applications such as a pendulum system, economic system, etc ${ }^{4) \sim 7)}$. The necessary and sufficient conditions for the controllability of discrete-time linear systems with positive controls were given by Evans and Murthy for single input case ${ }^{8)}$, and were generalized by Yoshida et al. to the multiple input case ${ }^{9)}$. But, regretfully it is not easy to determine whether a given multiple input system is positive controllable or not based on the former result ${ }^{9}$.

The purpose of this paper is to give a simple method to test the positive controllability of a multiple input discrete time linear systems.

\footnotetext{
* Department of Electrical and Electronics Engineering, Kagoshima University, 1-21-40, Korimoto, Kagoshima (Received March 27, 1998)

(Revised July 21, 1998)
}

\section{Preliminaries}

Consider a multiple input discrete-time linear system described by

$$
S: \boldsymbol{x}(k+1)=\boldsymbol{A} \boldsymbol{x}(k)+\boldsymbol{B} \boldsymbol{u}(k) ; k=0,1,2, \cdots
$$

where

$$
\begin{array}{ll}
\boldsymbol{A} \in \boldsymbol{R}^{n \times n}, & \boldsymbol{B} \in \boldsymbol{R}^{n \times m} \\
\boldsymbol{x}(k) \in \boldsymbol{R}^{n}, & \boldsymbol{u}(k) \in \boldsymbol{R}^{m}
\end{array}
$$

The control input is limited to the following condition;

$$
u_{i}(k) \geqq 0 ; i=1,2, \cdots, m
$$

where $u_{i}(k)$ is the $i$-th component of $\boldsymbol{u}(k)$.

Definition 1: The control input which satisfies (4) is called a positive control.

Definition 2: Let $\boldsymbol{x}(0)$ be any initial state. Then system $S$ is called to be positive controllable ( $p . c$.), if there exist a positive integer $\nu$ and a positive control sequence $\{\boldsymbol{u}(0)$, $\boldsymbol{u}(1), \cdots, \boldsymbol{u}(\nu-1)\}$ which will bring the system to the zero state from $x(0)$.

Definition 3: If $x_{i}>0$ or $x_{i} \geqq 0$ for all $i=1,2, \cdots, n$, then $\boldsymbol{x}=\left[x_{1}, x_{2}, \cdots, x_{n}\right]^{T}$ is called a positive vector $(\boldsymbol{x}>\boldsymbol{0})$, or a nonnegative vector $(\boldsymbol{x} \geqq \mathbf{0})$, respectively, where $T$ denotes the transpose.

Definition 4 : Let $\left\{k_{1}, k_{2}, \cdots, k_{m}\right\}$ be one of the rearrangements of $\{1,2, \cdots, m\}$. Then an $m \times m$ matrix $\boldsymbol{W}$ whose $i j$ element $w_{i j}$ is defined as

$$
\begin{aligned}
& w_{i j}=0 ; i \neq k_{j} ; j=1,2, \cdots, m \\
& w_{i j}=1 ; i=k_{j} ; j=1,2, \cdots, m
\end{aligned}
$$

is called an $m \times m$ permutation matrix.

\section{Controllability}

The positive controllability is invariant under any non- 
singular transformation of the state variable. Thus by a nonsingular real transformation matrix $\boldsymbol{U}$, we may describe the following Jordan canonical form for $\boldsymbol{A}$ and $\boldsymbol{B}$, over the field of real numbers:

$$
\begin{aligned}
& \boldsymbol{U}^{-1} \boldsymbol{A} \boldsymbol{U}=\text { block } \operatorname{diag}\left[\boldsymbol{A}_{1}, \boldsymbol{A}_{2}, \cdots, \boldsymbol{A}_{P},\right. \\
& \boldsymbol{A}_{P+1}, \boldsymbol{A}_{P+2}, \cdots, \boldsymbol{A}_{P+Q}, \\
& \left.\boldsymbol{A}_{P+Q+1}, \boldsymbol{A}_{P+Q+2}, \cdots, \boldsymbol{A}_{P+Q+R}\right] \\
& \in \boldsymbol{R}^{n \times n} \\
& \boldsymbol{U}^{-1} \boldsymbol{B}=\left[\boldsymbol{B}_{1}{ }^{T}, \boldsymbol{B}_{2}{ }^{T}, \cdots, \boldsymbol{B}_{P}{ }^{T},\right. \\
& \boldsymbol{B}_{P+1}{ }^{T}, \boldsymbol{B}_{P+2}{ }^{T}, \cdots, \boldsymbol{B}_{P+Q^{T}}, \\
& \left.\boldsymbol{B}_{P+Q+1}{ }^{T}, \boldsymbol{B}_{P+Q+2}{ }^{T}, \cdots, \boldsymbol{B}_{P+Q+R}{ }^{T}\right]^{T} \\
& \in \boldsymbol{R}^{n \times m} \\
& \boldsymbol{A}_{i}=\text { block } \operatorname{diag}\left[\boldsymbol{A}_{i 1}, \boldsymbol{A}_{i 2}, \cdots, \boldsymbol{A}_{i r(i)}\right] \\
& \in \boldsymbol{R}^{n(i) \times n(i)} ; i=1,2, \cdots, P+Q+R \\
& \boldsymbol{B}_{i}=\left[\boldsymbol{B}_{i 1}{ }^{T}, \boldsymbol{B}_{i 2}{ }^{T}, \cdots, \boldsymbol{B}_{i r(i)}{ }^{T}\right]^{T} \\
& \in \boldsymbol{R}^{n(i) \times m} ; i=1,2, \cdots, P+Q+R \\
& n=n(1)+n(2)+\cdots+n(P+Q+R) \\
& A_{i j}=\left[\begin{array}{cccccc}
\lambda_{i} & 1 & 0 & \cdots & 0 & 0 \\
0 & \lambda_{i} & 1 & \cdots & 0 & 0 \\
& \cdots & \cdots & & \cdots & \\
0 & 0 & \cdots & 0 & \lambda_{i} & 1 \\
0 & 0 & \cdots & 0 & 0 & \lambda_{i}
\end{array}\right], \\
& \in \boldsymbol{R}^{n(i, j) \times n(i, j)}, \quad \lambda_{i}>0 \text {; } \\
& i=1,2, \cdots, P ; j=1,2, \cdots, r(i) \\
& \boldsymbol{A}_{P+i, j}=\left[\begin{array}{cccccc}
\mu_{i} & 1 & 0 & \cdots & 0 & 0 \\
0 & \mu_{i} & 1 & \cdots & 0 & 0 \\
& \cdots & \cdots & & \cdots & \\
0 & 0 & \cdots & 0 & \mu_{i} & 1 \\
0 & 0 & \cdots & 0 & 0 & \mu_{i}
\end{array}\right] \text {, } \\
& \in \boldsymbol{R}^{n(P+i, j) \times n(P+i, j)}, \quad \mu_{i} \leqq 0 ; \\
& i=1,2, \cdots, Q ; j=1,2, \cdots, r(P+i) \\
& \boldsymbol{A}_{P+Q+i, j}=\left[\begin{array}{cccccc}
\boldsymbol{\Theta}_{i} & \boldsymbol{\Phi} & \mathbf{0} & \cdots & \mathbf{0} & 0 \\
\mathbf{0} & \boldsymbol{\Theta}_{i} & \boldsymbol{\Phi} & \cdots & \mathbf{0} & \mathbf{0} \\
& \cdots & & \cdots & \cdots & \\
\mathbf{0} & \mathbf{0} & \cdots & \mathbf{0} & \boldsymbol{\Theta}_{i} & \boldsymbol{\Phi} \\
\mathbf{0} & \mathbf{0} & \cdots & \mathbf{0} & \mathbf{0} & \boldsymbol{\Theta}_{i}
\end{array}\right] \\
& \in \boldsymbol{R}^{2 n(P+Q+i, j) \times 2 n(P+Q+i, j)} ; \\
& i=1,2, \cdots, R ; j=1,2, \cdots, r(P+Q+i) \\
& \boldsymbol{\Theta}_{i}=\left[\begin{array}{cc}
\sigma_{i} & \tau_{i} \\
-\tau_{i} & \sigma_{i}
\end{array}\right] ; \tau_{i}>0 ; i=1,2, \cdots, R \\
& \boldsymbol{\Phi}=\left[\begin{array}{ll}
0 & 1 \\
0 & 0
\end{array}\right] \\
& \boldsymbol{B}_{i j}=\left[\begin{array}{llll}
\boldsymbol{b}_{i j n(i, j)}{ }^{T}, & \cdots, & \boldsymbol{b}_{i j 2}{ }^{T}, & \boldsymbol{b}_{i j 1}{ }^{T}
\end{array}\right]^{T} \\
& \in \boldsymbol{R}^{n(i, j) \times m} \text {; } \\
& i=1,2, \cdots, P+Q ; j=1,2, \cdots, r(i) \\
& \boldsymbol{B}_{P+Q+i, j}=\left[\boldsymbol{B}_{i j n(i, j)}{ }^{T}, \cdots, \boldsymbol{B}_{i j 2}{ }^{T}, \quad \boldsymbol{B}_{i j 1}{ }^{T}\right]^{T} \\
& \in \boldsymbol{R}^{2 n(P+Q+i, j) \times m} \\
& i=1,2, \cdots, R ; j=1,2, \cdots, r(P+Q+i) \\
& n(i)=n(i, 1)+n(i, 2)+\cdots+n(i, r(i)) \text {; }
\end{aligned}
$$

$$
\begin{aligned}
& i=1,2, \cdots, P+Q \\
& n(i)=2 n(i, 1)+2 n(i, 2)+\cdots+2 n(i, r(i)) \\
& \quad i=P+Q+1, P+Q+2, \cdots, P+Q+R \\
& \boldsymbol{b}_{i j k} \in \boldsymbol{R}^{1 \times m} ; i=1,2, \cdots, P+Q ; \\
& j=1,2, \cdots, r(i) ; k=1,2, \cdots, n(i, j) \\
& \boldsymbol{B}_{i j k} \in \boldsymbol{R}^{2 \times m} ; i=1,2, \cdots, R ; \\
& j=1,2, \cdots, r(P+Q+i) ; k=1,2, \cdots, n(P+Q+i, j)
\end{aligned}
$$

where $\lambda_{i}(i=1,2, \cdots, P)$ are $P$ distinct positive eigenvalues, $\mu_{i}(i=1,2, \cdots, Q)$ are $Q$ distinct nonpositive eigenvalues, and $\sigma_{i} \pm \mathrm{j} \tau_{i}(i=1,2, \cdots, R)$ are $R$ distinct pairs of complex conjugate eigenvalues.

Then the following two theorems are known ${ }^{9}$.

Theorem 1: Assume that $\operatorname{rank}\left[\boldsymbol{B}, \boldsymbol{A B}, \cdots, \boldsymbol{A}^{n-1} \boldsymbol{B}\right]=n$. Then system $S$ is $p$. c . if and only if system $S_{i}{ }^{*}$ is $p$. c. for each $i=1,2, \cdots, P$, where

$$
\begin{aligned}
& S_{i}{ }^{*}: \boldsymbol{x}_{i}{ }^{*}(k+1)=\lambda_{i} \boldsymbol{x}_{i}{ }^{*}(k)+\boldsymbol{B}_{i}{ }^{*} \boldsymbol{u}(k), \lambda_{i}>0 \\
& \boldsymbol{x}_{i}{ }^{*}(k) \in \boldsymbol{R}^{r(i)} \\
& \boldsymbol{B}_{i}{ }^{*}=\left[\boldsymbol{b}_{i 11}{ }^{T}, \boldsymbol{b}_{i 21}{ }^{T}, \cdots, \boldsymbol{b}_{i r(i) 1}{ }^{T}\right]^{T} \in \boldsymbol{R}^{r(i) \times m}
\end{aligned}
$$

Theorem 2: System $S^{*}$ described by

$$
S^{*}: \boldsymbol{x}^{*}(k+1)=\lambda \boldsymbol{x}^{*}(k)+\boldsymbol{B}^{*} \boldsymbol{u}(k), \quad \lambda>0
$$

where

$$
\begin{aligned}
& \boldsymbol{x}^{*}(k) \in \boldsymbol{R}^{F}, \quad \boldsymbol{u}(k) \in \boldsymbol{R}^{m} \\
& \boldsymbol{B}^{*} \triangleq\left[\boldsymbol{\beta}_{1}, \boldsymbol{\beta}_{2}, \cdots, \boldsymbol{\beta}_{m}\right] \in \boldsymbol{R}^{F \times m}
\end{aligned}
$$

is $p$. c. if and only if there exist positive integers $\left\{h, K_{1}, K_{2}\right.$, $\left.\cdots, K_{h}\right\}$ and positive scalars $\left\{C_{K_{1}}, C_{K_{2}}, \cdots, C_{K_{h}}\right\}$ which satisfy the following three conditions;

(1) $c_{K_{1}} \boldsymbol{\beta}_{K_{1}}+c_{K_{2}} \boldsymbol{\beta}_{K_{2}}+\cdots+c_{K_{h}} \boldsymbol{\beta}_{K_{h}}=\mathbf{0}$

(2) $1 \leqq K_{1}<K_{2}<\cdots<K_{h} \leqq m$

(3) $\operatorname{rank}\left[\boldsymbol{\beta}_{K_{1}}, \boldsymbol{\beta}_{K_{2}}, \cdots, \boldsymbol{\beta}_{K_{h}}\right]=F<h \leqq m$

Remark 1: If system $S^{*}$ is $p$. c. then

$$
\operatorname{rank} \boldsymbol{B}^{*}=F<m
$$

In Theorem 2, the suffix $i$ for system $S_{i}{ }^{*}$ is neglected in order to avoid the complexity of the notations. Hereafter we delete the suffix $i$ for system $S_{i}{ }^{*}$. Furthermore from Theorem 2, it is evident that the positive controllability of system $S^{*}$ depends on matrix $\boldsymbol{B}^{*}$ only. Thus for the remainder of this paper, system $S^{*}$ will also be called system $\boldsymbol{B}^{*}$ for convenience.

Although Theorem 2 presents a necessary and sufficient condition for positive controllability, it is not easy to find out the positive integer and positive scalars which satisfy (29) $\sim(31)$. Thus, in what follows, a simple method to test the positive controllability will be proposed.

\section{Controllability Test}

At first we obtain the following lemma easily.

Lemma 1: Assume that $\boldsymbol{B}^{*}$ is expressed as 


$$
B^{*}=\left[G_{1}, G_{2}\right]
$$

where

$$
\begin{aligned}
& \boldsymbol{G}_{1} \in \boldsymbol{R}^{F \times m_{1}}, \quad \boldsymbol{G}_{2} \in \boldsymbol{R}^{F \times m_{2}} \\
& m=m_{1}+m_{2}
\end{aligned}
$$

Then system $\boldsymbol{B}^{*}$ is $p$. c. if system $\boldsymbol{G}_{1}$ or system $\boldsymbol{G}_{2}$ is $p$. c.

Next we obtain the following theorem.

Theorem 3: System $\boldsymbol{B}^{*}$ is $p$. c. if and only if $\operatorname{rank} \boldsymbol{B}^{*}=$ $F$ and there exists a positive vector $\Omega^{*}$ such that

$$
\boldsymbol{B}^{*} \boldsymbol{\Omega}^{*}=\mathbf{0}, \boldsymbol{\Omega}^{*} \triangleq\left[\omega_{1}, \omega_{2}, \cdots, \omega_{m}\right]^{T}>\mathbf{0}
$$

The proof is given in Appendix A.

Now let $\boldsymbol{W}$ be an $m \times m$ permutation matrix and $\boldsymbol{V}$ be an $F \times F$ nonsingular real matrix. Next consider system $S^{*}$ described by

$$
S^{*}: \boldsymbol{x}^{*}(k+1)=\lambda \boldsymbol{x}^{*}(k)+\boldsymbol{B}^{*} \boldsymbol{u}^{*}(k), \quad \lambda>0
$$

where

$$
\begin{aligned}
& \boldsymbol{B}^{\#} \triangleq \boldsymbol{V}^{-1} \boldsymbol{B}^{*} \boldsymbol{W} \\
& \boldsymbol{x}^{\#}(k) \triangleq \boldsymbol{V}^{-1} \boldsymbol{x}^{*}(k) \in \boldsymbol{R}^{F} \\
& \boldsymbol{u}^{\#}(k) \triangleq \boldsymbol{W}^{-1} \boldsymbol{u}(k) \in \boldsymbol{R}^{m}
\end{aligned}
$$

Then $\boldsymbol{x}(k)$ and $\boldsymbol{x}^{\sharp}(k)$ correspond to each other, from (39). Further a positive control $\boldsymbol{u}(k)$ and a positive control $\boldsymbol{u}^{\sharp}(k)$ correspond to each other, from (40). Thus by definition 2 , the following theorem is established.

Theorem 4 : System $\boldsymbol{B}^{*}$ is $p$. c. if and only if system $\boldsymbol{B}^{*}$ $=\boldsymbol{V}^{-1} \boldsymbol{B}^{*} \boldsymbol{W}$ is $p$. c. where $\boldsymbol{W}$ is an $m \times m$ permutation matrix and $\boldsymbol{V}$ is an $F \times F$ nonsingular real matrix.

The following theorem is straightforward from Theorem 3 and Theorem 4.

Theorem 5: System $\boldsymbol{B}^{*}$ is $p$. c. if and only if rank $\boldsymbol{B}^{*}=$ $F$ and there exists a positive vector $\Omega^{*}$. such that

$$
\boldsymbol{B}^{\sharp} \boldsymbol{\Omega}^{\#}=\mathbf{0}, \boldsymbol{\Omega}^{\#} \triangleq\left[\omega^{\#}{ }_{1}, \omega^{\#}{ }_{2}, \cdots, \omega_{m}^{\#}\right]^{T}>\mathbf{0}
$$

Remark 2: If rank $\boldsymbol{B}^{*}=F$, then there exist some $m \times m$ permutation matrix $\boldsymbol{W}$ and some $F \times F$ nonsingular real matrix $V$ such that

$$
\boldsymbol{B}^{*}=\boldsymbol{V}^{-1} \boldsymbol{B}^{*} \boldsymbol{W}=\left[\boldsymbol{I}_{F}, \boldsymbol{G}\right] ; \boldsymbol{G} \in \boldsymbol{R}^{F \times(m-F)}
$$

The total number to select a set of $F$ independent vectors out of $m$ vectors is at most ${ }_{m} \mathrm{C}_{F}$. Thus the number of matrices, $\boldsymbol{W}$ and $\boldsymbol{V}$, which satisfy (42) is at most ${ }_{m} \mathrm{C}_{F}$. $\square$

When $F=1$, we obtain the following lemma.

Lemma 2 : When $F=1$ and $m>1$, system $\boldsymbol{B}^{*}$ is $p$. c. if and only if there exist an $m \times m$ permutation matrix $\boldsymbol{W}$, a nonzero real scalar $V$, and a positive scalar $g$ such that

$$
\boldsymbol{B}^{\sharp}=V^{-1} \boldsymbol{B}^{*} \boldsymbol{W}=[1,-g, *], g>0
$$

where $*$ denotes $1 \times(m-2)$ real matrix.

The proof is omitted.

When $F=m-1$, we obtain the following lemma.

Lemma 3 : When $F=m-1$ and $m>1$, system $\boldsymbol{B}^{*}$ is $p$. $c$. if and only if there exist an $m \times m$ permutation matrix $\boldsymbol{W}$, an $(m-1) \times(m-1)$ nonsingular real matrix $\boldsymbol{V}$, and an $(m$
$-1) \times 1$ positive vector $\boldsymbol{g}$ such that

$$
\boldsymbol{B}^{\sharp}=\boldsymbol{V}^{-1} \boldsymbol{B}^{*} \boldsymbol{W}=\left[\boldsymbol{I}_{m-1},-\boldsymbol{g}\right], \boldsymbol{g}>\mathbf{0}
$$

The proof is given in Appendix B.

When $m=2$, Lemma 2 is equivalent to Lemma 3 .

Next we obtain the following lemmas.

Lemma 4: Assume that

$$
\boldsymbol{B}^{*}=\boldsymbol{V}^{-1} \boldsymbol{B}^{*} \boldsymbol{W}=\left[\boldsymbol{I}_{F}, \boldsymbol{G}^{-}, \boldsymbol{g}\right]
$$

where $\boldsymbol{G}^{-}$is an $F \times(m-F-1)$ real matrix and $\boldsymbol{g} \triangleq\left[g_{1}, g_{2}\right.$, $\left.\cdots, g_{F}\right]^{T} \geqq 0$ is an $F \times 1$ nonnegative vector. Then system $\boldsymbol{B}^{*}$ is $p$. c. if and only if system $\boldsymbol{B}^{-}$is $p$. c., where

$$
\boldsymbol{B}^{-} \triangleq\left[\boldsymbol{I}_{F}, \boldsymbol{G}^{-}\right] \in \boldsymbol{R}^{F \times(m-1)}
$$

The proof is given in Appendix C.

Lemma 5: Assume that an $F \times m$ real matrix $\boldsymbol{B}^{\#}$ is subdivided into

$$
\boldsymbol{B}^{\sharp}=\left[\begin{array}{ccc}
\boldsymbol{I}_{F_{1}} & \mathbf{0} & \boldsymbol{G}_{1} \\
\mathbf{0} & \boldsymbol{I}_{F_{2}} & \boldsymbol{G}_{2}
\end{array}\right]
$$

where

$$
\begin{aligned}
& \boldsymbol{G}_{i} \in \boldsymbol{R}^{F_{i} \times(m-F)} ; i=1,2 \\
& F=F_{1}+F_{2}
\end{aligned}
$$

Then two systems $\boldsymbol{B}^{\#}{ }_{1}$ and $\boldsymbol{B}^{{ }_{2}}{ }_{2}$ are $p$. c., where

$$
\boldsymbol{B}^{*}{ }_{i} \triangleq\left[\boldsymbol{I}_{F_{i}}, \boldsymbol{G}_{i}\right] \in \boldsymbol{R}^{F_{\iota} \times\left(F_{i}+m-F\right)}
$$

if system $\boldsymbol{B}^{*}$ is $p . c$.

The proof is omitted.

Remark 3 : If $\boldsymbol{B}^{*}$ has a row whose elements are all nonnegative, then system $S^{*}$ is not $p$. c. from Lemma 2 and Lemma 5.

Furthermore we obtain the following theorem which contains Lemma 2 and Lemma 3 as special cases.

Theorem 6 : When $\boldsymbol{B}^{*}$ is an $F \times m$ real matrix and $F<$ $m$, system $\boldsymbol{B}^{*}$ is $p$. c. if and only if there exist positive integers $\left\{s, F_{1}, F_{2}, \cdots, F_{s}\right\}$, an $m \times m$ permutation matrix $\boldsymbol{W}$, an $F \times F$ nonsingular real matrix $\boldsymbol{V}$, and $F_{i} \times 1$ positive vectors $\boldsymbol{g}_{i} ;(i=1,2, \cdots, s)$ such that

$$
\boldsymbol{B}^{*}=\boldsymbol{V}^{-1} \boldsymbol{B}^{*} \boldsymbol{W}=\left[\boldsymbol{I}_{F},-\boldsymbol{G}, *\right]
$$

where $\boldsymbol{G}$ is an $F \times s$ real matrix defined as

$$
\begin{array}{r}
\boldsymbol{G} \triangleq\left[\begin{array}{ccccc}
\boldsymbol{g}_{1} & * & * & \cdots & * \\
\mathbf{0} & \boldsymbol{g}_{2} & * & \cdots & * \\
& \cdots & \cdots & & \cdots \\
\mathbf{0} & \mathbf{0} & \cdots & \mathbf{0} & \boldsymbol{g}_{s}
\end{array}\right] \\
1 \leqq s \leqq F_{1}+F_{2}+\cdots+F_{s}=F
\end{array}
$$

with $*$ denoting some real matrix of appropriate size. $\square$

The proof is given in Appendix D.

Summarizing Remark 1, Remark 2, Remark 3, Lemma 4, and Theorem 6 , the positive controllability test may be checked in a finite number of trials by applying the elimination method of Gauss. 


\section{Numerical Example}

Consider a system $S$ represented by

$$
\begin{aligned}
\boldsymbol{U}^{-1} \boldsymbol{A} \boldsymbol{U} & =\left[\begin{array}{lllllll}
2 & 0 & 0 & 0 & 0 & 0 & 0 \\
0 & 2 & 0 & 0 & 0 & 0 & 0 \\
0 & 0 & 2 & 1 & 0 & 0 & 0 \\
0 & 0 & 0 & 2 & 0 & 0 & 0 \\
0 & 0 & 0 & 0 & -1 & 0 & 0 \\
0 & 0 & 0 & 0 & 0 & 3 & 4 \\
0 & 0 & 0 & 0 & 0 & -4 & 3
\end{array}\right] \\
\boldsymbol{U}^{-1} \boldsymbol{B} & =\left[\begin{array}{rrrrrr}
2 & -2 & -1 & 1 & -4 & 1 \\
1 & 1 & 0 & 1 & 3 & -2 \\
1 & 0 & -1 & 4 & -2 & 3 \\
1 & -2 & 1 & 1 & -3 & 1 \\
-1 & 0 & 3 & 0 & 1 & 0 \\
0 & 2 & 2 & -1 & 5 & 4 \\
3 & -1 & 1 & 0 & 2 & 1
\end{array}\right]
\end{aligned}
$$

where

$$
n=7, \quad m=6, \quad P=1, \quad Q=1, \quad R=1
$$

Then we have

$$
\begin{aligned}
& \boldsymbol{A}_{1}=\left[\begin{array}{llll}
2 & 0 & 0 & 0 \\
0 & 2 & 0 & 0 \\
0 & 0 & 2 & 1 \\
0 & 0 & 0 & 2
\end{array}\right], \quad A_{2}=-1 \\
& A_{3}=\left[\begin{array}{rr}
3 & 4 \\
-4 & 3
\end{array}\right]=\Theta_{1} \\
& \boldsymbol{B}_{1}=\left[\begin{array}{rrrrrr}
2 & -2 & -1 & 1 & -4 & 1 \\
1 & 1 & 0 & 1 & 3 & -2 \\
1 & 0 & -1 & 4 & -2 & 3 \\
1 & -2 & 1 & 1 & -3 & 1
\end{array}\right] \\
& \boldsymbol{B}_{2}=\left[\begin{array}{llllll}
-1 & 0 & 3 & 0 & 1 & 0
\end{array}\right] \\
& \boldsymbol{B}_{3}=\left[\begin{array}{rrrrrr}
0 & 2 & 2 & -1 & 5 & 4 \\
3 & -1 & 1 & 0 & 2 & 1
\end{array}\right] \\
& \boldsymbol{A}_{11}=\boldsymbol{A}_{12}=2, \quad \boldsymbol{A}_{13}=\left[\begin{array}{ll}
2 & 1 \\
0 & 2
\end{array}\right] \\
& \boldsymbol{B}_{11}=\left[\begin{array}{llllll}
2 & -2 & -1 & 1 & -4 & 1
\end{array}\right]=\boldsymbol{b}_{111} \\
& \boldsymbol{B}_{12}=\left[\begin{array}{llllll}
1 & 1 & 0 & 1 & 3 & -2
\end{array}\right]=\boldsymbol{b}_{121} \\
& \boldsymbol{B}_{13}=\left[\begin{array}{rrrrrr}
1 & 0 & -1 & 4 & -2 & 3 \\
1 & -2 & 1 & 1 & -3 & 1
\end{array}\right]=\left[\begin{array}{l}
\boldsymbol{b}_{132} \\
\boldsymbol{b}_{131}
\end{array}\right]
\end{aligned}
$$

where

$$
\begin{aligned}
& n(1)=4, \quad n(2)=1, \quad n(3)=2 \\
& r(1)=3, \quad r(2)=1, \quad r(3)=1 \\
& n(1,1)=1, \quad n(1,2)=1, \quad n(1,3)=2 \\
& n(2,1)=1, \quad n(3,1)=1 \\
& \lambda_{1}=2, \quad \mu_{1}=-1, \quad \sigma_{1}=3, \quad \tau_{1}=4
\end{aligned}
$$

Thus we get

$$
\boldsymbol{B}^{*}=\left[\begin{array}{rrrrrr}
2 & -2 & -1 & 1 & -4 & 1 \\
1 & 1 & 0 & 1 & 3 & -2 \\
1 & -2 & -1 & 1 & -3 & 1
\end{array}\right]
$$

$$
\begin{aligned}
\quad=\left[\begin{array}{llllll}
\boldsymbol{\beta}_{1} & \boldsymbol{\beta}_{2} & \boldsymbol{\beta}_{3} & \boldsymbol{\beta}_{4} & \boldsymbol{\beta}_{5} & \boldsymbol{\beta}_{6}
\end{array}\right] \\
F=3, \quad m=6
\end{aligned}
$$

At the first trial, choosing $W$ and $V$ as

$$
\begin{aligned}
& \boldsymbol{W}=\boldsymbol{I}_{6} \\
& \boldsymbol{V}=\left[\begin{array}{rrr}
2 & -2 & -1 \\
1 & 1 & 0 \\
1 & -2 & -1
\end{array}\right]
\end{aligned}
$$

then we have

$$
\begin{aligned}
\boldsymbol{B}^{*} & =\boldsymbol{V}^{-1} \boldsymbol{B}^{*} \boldsymbol{W} \\
& =\left[\begin{array}{rrrrrr}
1 & 0 & 0 & 0 & -1 & 0 \\
0 & 1 & 0 & 1 & 4 & -2 \\
0 & 0 & 1 & -3 & -6 & 3
\end{array}\right]
\end{aligned}
$$

Thus we can not succeed in obtaining the form of equations (51) and (52).

Next for another trial, choosing $\boldsymbol{W}$ and $\boldsymbol{V}$ as

$$
\begin{aligned}
& \boldsymbol{W}=\left[\begin{array}{llllll}
0 & 0 & 1 & 0 & 0 & 0 \\
1 & 0 & 0 & 0 & 0 & 0 \\
0 & 0 & 0 & 0 & 0 & 1 \\
0 & 1 & 0 & 0 & 0 & 0 \\
0 & 0 & 0 & 0 & 1 & 0 \\
0 & 0 & 0 & 1 & 0 & 0
\end{array}\right] \\
& \boldsymbol{V}=\left[\begin{array}{rrr}
-2 & 1 & 2 \\
1 & 1 & 1 \\
-2 & 1 & 1
\end{array}\right]
\end{aligned}
$$

then we obtain

$$
\begin{aligned}
\boldsymbol{B}^{*} & =\boldsymbol{V}^{-1} \boldsymbol{B}^{*} \boldsymbol{W} \\
& =\left[\begin{array}{lllrrr}
1 & 0 & 0 & -1 & 2 & 0.333 \\
0 & 1 & 0 & -1 & 2 & -0.333 \\
0 & 0 & 1 & 0 & -1 & 0
\end{array}\right] \\
s & =2, \quad F_{1}=2, \quad F_{2}=1, \quad \boldsymbol{g}_{1}=[1, \quad 1]^{T}, \quad \boldsymbol{g}_{2}=1
\end{aligned}
$$

Thus system $S^{*}$ is $p$. c. from Theorem 6 . Therefore, system $S$ is $p$. c. from Theorem 1 .

\section{Conclusions}

Simple criteria for multiple input discrete-time linear systems to become positive controllable are given based on the Jordan canonical form and elimination method of Gauss. It is pointed out that the number of steps necessary to discriminate the positive controllability is finite. The results presented here clarify the structure of positive controllable systems and are useful in discussing fundamental control problems.

\section{References}

1) R.E. Kalman, Y.C. Ho, and K.S. Narendra: Controllability of Linear Dynamical Systems, Contributions to J. Differential Equations, 1, 189/213(1962)

2) J. Wing and C.A. Desoer: The multiple-input minimal time regulator problem(general theory), IEEE Trans. Automat. Contr., AC-5, 125/136 (1963)

3) S. Kodama : Controllability of Linear Discrete-Time Sys- 
tems with Input and State Variable Constraints, J. Inst. Elec. Comm. Engrs., 48-5, 884/891 (1965)

4) S. H. Saperstone and J. A. Yorke: Controllability of Linear Oscillatory Systems Using Positive Controls, SIAM J. Contr., 9-2, 253/262 (1971)

5) R. F. Bramer: Controllability in Linear Autonomous Systems with Positive Controllers, SIAM J. Contr., 10-2, 339/ 353(1972)

6) S. H. Saperstone: Global Controllability of Linear systems with Positive Controls, SIAM J. Contr., 11-3, 417/ 423(1973)

7) H. Kusuoka, H. Maeda, and S. Kodama: On the Reachability of Discrete-Time Compartment Systems with Nonnegative Input Constraints, J. Inst. Elec. Comm. Engrs., 63 -A-2, 67/73 (1980)

8) M.E. Evans and D. N.P. Murthy: Controllability of Discrete-Time Systems with Positive Controls, IEEE Trans. Automat. Contr., 22, 942/945(1977)

9) H. Yoshida, Y. Tanada, T. Tanaka, and K. Yunokuchi : Controllability of Multiple Input Discrete-Time Linear Systems with Positive Controls, Trans. SICE, 30-3, 243/ 252(1994)

\section{Appendix A : Proof of Theorem 3}

Sufficiency : Assume that $\operatorname{rank} \boldsymbol{B}^{*}=F$ and there exist a positive vector $\boldsymbol{\Omega}^{*}$ such that (36) holds. Then (29) $\sim(31)$ hold by choosing

$$
h=m, \quad K_{i}=i, \quad c_{K_{i}}=\omega_{i} ; i=1,2, \cdots, m .
$$

Thus from Theorem 2, $\boldsymbol{B}^{*}$ is $p . c$.

Necessity: If $\boldsymbol{B}^{*}$ is $p$. c., then from Theorem 2 there exist positive integers $\left\{h, K_{1}, K_{2}, \cdots, K_{h}\right\}$ and positive scalars $\left\{c_{K_{1}}, c_{K_{2}}, \cdots, c_{K_{n}}\right\}$ which satisfy $(29) \sim(31)$.

Now we can select $F$ linearly independent vectors

$$
\left\{\boldsymbol{\beta}_{N 1}, \boldsymbol{\beta}_{N 2}, \cdots, \boldsymbol{\beta}_{N_{F}}\right\}
$$

out of $\left\{\boldsymbol{\beta}_{K 1}, \boldsymbol{\beta}_{K 2}, \cdots, \boldsymbol{\beta}_{K n}\right\}$ in (31), where $1 \leqq N_{1}<N_{2}<\cdots<N_{F}$ $\leqq m$. Next we let

$$
\boldsymbol{\beta} \triangleq-\boldsymbol{\beta}_{1}-\boldsymbol{\beta}_{2}-\cdots-\boldsymbol{\beta}_{m} \in \boldsymbol{R}^{F}
$$

Then $\beta$ can be expressed by the $F$ linearly independent vectors in (A.1). Thus we have

$$
\begin{aligned}
& \boldsymbol{\beta}_{1}+\boldsymbol{\beta}_{2}+\cdots+\boldsymbol{\beta}_{m} \\
& \quad+d_{N_{1}} \beta_{N_{1}}+d_{N_{2}} \beta_{N_{2}}+\cdots+d_{N_{F}} \beta_{N_{F}}=\mathbf{0}
\end{aligned}
$$

Premultiplying both sides of (29) by a sufficiently large positive scalar $M$ and adding (A.3) yield

$$
\omega^{*}{ }_{1} \boldsymbol{\beta}_{1}+\omega^{*}{ }_{2} \boldsymbol{\beta}_{2}+\cdots+\omega^{\#}{ }_{m} \boldsymbol{\beta}_{m}=\mathbf{0}
$$

where

$$
\begin{aligned}
& \omega^{*}{ }_{i} \triangleq 1+M c_{i}+d_{i}>0 ; i=N_{1}, N_{2}, \cdots, N_{F} \\
& \omega^{*}{ }_{i} \triangleq 1+M c_{i}>0 \quad ; i=K_{1}, K_{2}, \cdots, K_{h} \\
& \quad \text { and } \quad i \neq N_{1}, N_{2}, \cdots, N_{F} \\
& \omega^{*}{ }_{i} \triangleq 1>0 ; i=1,2, \cdots, m \text { and } i \neq K_{1}, K_{2}, \cdots, K_{h}
\end{aligned}
$$

Thus there exists a positive vector $\Omega^{*}$ such that

$$
\boldsymbol{B}^{*} \boldsymbol{\Omega}^{*}=\mathbf{0}, \boldsymbol{\Omega}^{*} \triangleq\left[\boldsymbol{\omega}_{1}^{\#}, \boldsymbol{\omega}^{\#}{ }_{2}, \cdots, \boldsymbol{\omega}_{m}^{*}\right]^{T}>\mathbf{0}
$$

\section{Appendix B : Proof of Lemma 3}

Necessity: Assume that system $\boldsymbol{B}^{*}$ is $p$. c.. Then rank $\boldsymbol{B}^{*}=m-1$ by Remark 1 . Furthermore by Remark 2 , there exist an $m \times m$ permutation matrix $\boldsymbol{W}$ and an $(m-1) \times(m$ -1) nonsingular real matrix $\boldsymbol{V}$ such that

$$
\boldsymbol{B}^{\sharp}=\boldsymbol{V}^{-1} \boldsymbol{B}^{*} \boldsymbol{W}=\left[\boldsymbol{I}_{m-1},-\boldsymbol{g}\right]
$$

where

$$
\boldsymbol{g} \triangleq\left[g_{1}, g_{2}, \cdots, g_{m-1}\right]^{T}
$$

On the other hand, from Theorem 5 there exists a positive vector $\boldsymbol{\Omega}^{*}$ such that

$$
\begin{aligned}
& \boldsymbol{\Omega}^{\#} \triangleq\left[\boldsymbol{\omega}_{1}^{\#}, \boldsymbol{\omega}^{\#}{ }_{2}, \cdots, \boldsymbol{\omega}^{\#}{ }_{m}\right]^{T} ; \boldsymbol{\omega}^{\#}{ }_{i}>0 ; \\
& \quad i=1,2, \cdots, m \\
& \boldsymbol{B}^{\#} \boldsymbol{\Omega}^{\#}=\mathbf{0}
\end{aligned}
$$

since system $\boldsymbol{B}^{*}$ is assumed to be p. c.. Substituting (B.1) $\sim$ (B.3) for (B.4), we have

$$
\omega^{*}{ }_{i}-g_{i} \omega^{*}{ }_{m}=0 ; i=1,2, \cdots, m-1
$$

From (B.3) and (B.5), it is derived that

$$
g_{i}>0 ; \quad i=1,2, \cdots, m-1
$$

Thus $\boldsymbol{g}$ in (B.1) is a positive vector, i. e. $\boldsymbol{g}>\mathbf{0}$. Therefore we obtain (44).

Sufficiency: Suppose that $\boldsymbol{B}^{\#}$ is represented by (44). Then rank $\boldsymbol{B}^{\sharp}=m-1$. Further letting $\boldsymbol{\Omega}^{\#} \triangleq\left[\boldsymbol{g}^{T}, 1\right]^{T}$, we get

$$
\boldsymbol{B}^{\sharp} \boldsymbol{\Omega}^{\sharp}=\mathbf{0} ; \boldsymbol{\Omega}^{\sharp}>\mathbf{0}
$$

Thus system $\boldsymbol{B}^{*}$ is $p$. c. by Theorem 5 .

Q.E.D.

\section{Appendix C : Proof of Lemma 4}

Sufficiency: Suppose that system $\boldsymbol{B}^{-1}$ is $p$.c.. Then system $\boldsymbol{B}^{\#}$ is $p$. c. by Lemma 1 . Thus system $\boldsymbol{B}^{*}$ is $p$. c. by Theorem 4.

Necessity: Assume that system $\boldsymbol{B}^{*}$ is $p$. $c$.. Then from Theorem 5 there exists a positive vector $\boldsymbol{\Omega}^{\#}$ such that

$$
\boldsymbol{B}^{*} \boldsymbol{\Omega}^{\#}=\mathbf{0}, \boldsymbol{\Omega}^{*} \triangleq\left[\omega^{\#}{ }_{1}, \omega^{\#}{ }_{2}, \cdots, \omega^{\#}\right]^{T}>\mathbf{0}
$$

Now considering (45), let

$$
\boldsymbol{B}^{\#} \triangleq\left[\boldsymbol{I}_{F}, \boldsymbol{G}^{-}, \boldsymbol{g}\right] \triangleq\left[\boldsymbol{\beta}^{\#}{ }_{1}, \boldsymbol{\beta}^{\#}{ }_{2}, \cdots, \boldsymbol{\beta}^{*}{ }_{m}\right]
$$

Then we obtain from (45), (46) and (C.2)

$$
\begin{aligned}
& \boldsymbol{B}^{-}=\left[\boldsymbol{I}_{F}, \boldsymbol{G}^{-}\right]=\left[\boldsymbol{\beta}^{*}{ }_{1}, \boldsymbol{\beta}^{*}{ }_{2}, \cdots, \boldsymbol{\beta}^{*}{ }_{m-1}\right] \\
& \operatorname{rank} \boldsymbol{B}^{-}=F
\end{aligned}
$$

Furthermore, postmultiplying both sides of (C.2) by $\left[\boldsymbol{g}^{T}, \mathbf{0},-1\right]^{T}$ we have

$$
\mathbf{0}=g_{1} \boldsymbol{\beta}^{*}{ }_{1}+g_{2} \boldsymbol{\beta}^{\#}{ }_{2}+\cdots+g_{F} \boldsymbol{\beta}^{\#}{ }_{F}-\boldsymbol{\beta}^{\#}{ }_{m}
$$

Thus from (C.1) (C.3) and (C.5) we have

$$
\begin{aligned}
& \omega^{\#}{ }_{1} \beta^{\#}{ }_{1}+\omega^{\#}{ }_{2} \beta^{\#}{ }_{2}+\cdots+\omega^{\#}{ }_{m} \beta^{\#}{ }_{m} \\
& \quad=\omega^{+}{ }_{1} \boldsymbol{\beta}^{\#}{ }_{1}+\omega^{+}{ }_{2} \beta^{*}{ }_{2}+\cdots+\omega^{+}{ }_{m-1} \beta^{\#}{ }_{m-1}=\mathbf{0}
\end{aligned}
$$

where

$$
\begin{aligned}
& \omega^{+}{ }_{i} \triangleq \omega^{\#}{ }_{i}+g_{i} \omega^{\#}{ }_{m}>0 ; i=1,2, \cdots, F \\
& \omega^{+}{ }_{i} \triangleq \omega^{\#}{ }_{i}>0 ; i=F+1, F+2, \cdots, m-1
\end{aligned}
$$


If we let

$$
\boldsymbol{\Omega}^{+}=\left[\omega^{+}{ }_{1}, \omega^{+}{ }_{2}, \cdots, \omega^{+}{ }_{m-1}\right]^{T}
$$

then from (C.3) and (C.6) (C.9), we obtain

$$
B^{-} \boldsymbol{\Omega}^{+}=\mathbf{0}, \quad \boldsymbol{\Omega}^{+}>0
$$

Therefore system $\boldsymbol{B}^{-}$is p.c. from Theorem 3. Q.E.D.

\section{Appendix D : Proof of Theorem 6}

Sufficiency: Suppose that $(51) \sim(53)$ hold. Then it is easy to show that rank $\boldsymbol{B}^{*}=F$, and there exist a positive vector $\boldsymbol{\Omega}^{*} \triangleq\left[\omega^{\#}{ }_{1}, \omega^{\#}{ }_{2}, \cdots, \omega_{m}^{\#}\right]^{T}$ such that

$$
B^{\#} \Omega^{*}=0, \quad \Omega^{*}>0
$$

by choosing

$$
\begin{aligned}
& \omega^{\#}{ }_{F+1} \gg \omega_{F+2}^{\#} \gg \cdots \gg \omega_{F+s}^{\#} \gg 1, \\
& \omega^{\#}{ }_{F+s+1}=\omega^{\#}{ }_{F+s+2}=\cdots=\omega^{*}{ }_{m}=1
\end{aligned}
$$

Thus system $\boldsymbol{B}^{*}$ is $p$. c. by Theorem 5 .

Necessity: When $F=1$, necessity is valid from Lemma 2.

Next, we assume that necessity is valid when $F=1,2, \cdots$, $L$, for all positive integer $m$, such that $1 \leqq L \leqq m-1$. Under this hypothesis we will show that necessity is valid when $F=L+1$.

Now assume that system $\widetilde{\boldsymbol{B}}$ is $p$. c., where

$$
\tilde{\boldsymbol{B}} \in \boldsymbol{R}^{(L+1) \times m}
$$

Then $\operatorname{rank} \widetilde{\boldsymbol{B}}=L+1<m$, from Remark 1. Thus from Remark 2, there exist an $m \times m$ permutation matrix $\boldsymbol{W}_{1}$ and an $(L+1) \times(L+1)$ nonsingular real matrix $\boldsymbol{V}_{\mathbf{1}}$, such that

$$
\widetilde{\boldsymbol{B}}_{2} \triangleq\left(\boldsymbol{V}_{1}\right)^{-1} \widetilde{\boldsymbol{B}} \boldsymbol{W}_{1}=\left[\begin{array}{ccc}
\boldsymbol{I}_{L} & \mathbf{0} & \boldsymbol{G}_{1} \\
\mathbf{0} & 1 & \boldsymbol{G}_{2}
\end{array}\right]
$$

where $\boldsymbol{G}_{1}$ is an $L \times(m-L-1)$ real matrix, and $\boldsymbol{G}_{2}$ is an 1 $\times(m-L-1)$ real matrix. Since system $\widetilde{\boldsymbol{B}}$ is $p$. c., system $\boldsymbol{B}^{\#}{ }_{1}$ is $p$. c. from Lemma 5 , where

$$
\boldsymbol{B}^{\#}{ }_{1} \triangleq\left[\boldsymbol{I}_{L}, \quad \boldsymbol{G}_{1}\right] \in \boldsymbol{R}^{L \times(m-1)}
$$

Thus from the hypothesis, there exist positive integers $\left\{t, L_{1}, L_{2}, \cdots, L_{t}\right\}$, an $(m-1) \times(m-1)$ permutation matrix $\boldsymbol{W}_{2}$, an $L \times L$ nonsingular real matrix $\boldsymbol{V}_{2}$, and $L_{i} \times 1$ positive vectors $\boldsymbol{y}_{i} ;(i=1,2, \cdots, t)$ such that

$$
\left(\boldsymbol{V}_{2}\right)^{-1} \boldsymbol{B}^{\#}{ }_{1} \boldsymbol{W}_{2}=\left[\begin{array}{lll}
\boldsymbol{I}_{L}, & -\boldsymbol{Y}, & *
\end{array}\right]
$$

where $Y$ is an $L \times t$ real matrix defined as

$$
\begin{aligned}
& Y \triangleq\left[\begin{array}{ccccc}
\boldsymbol{y}_{1} & * & * & \cdots & * \\
0 & \boldsymbol{y}_{2} & * & \cdots & * \\
\cdots & & \cdots & \cdots & \\
0 & 0 & \cdots & 0 & \boldsymbol{y}_{t}
\end{array}\right] \\
& 1 \leqq t \leqq L_{1}+L_{2}+\cdots+L_{t}=L
\end{aligned}
$$

with $*$ denoting some real matrix of appropriate size.

Now let

$\left[0, G_{2}\right] W_{2} \triangleq[\alpha, *], \alpha \in R^{1 \times L}$

$$
\begin{aligned}
& \boldsymbol{W}_{3} \triangleq\left[\begin{array}{ccc}
\mathbf{0} & \boldsymbol{I}_{L} & \mathbf{0} \\
1 & \mathbf{0} & \mathbf{0} \\
\mathbf{0} & \mathbf{0} & \boldsymbol{I}_{m-L-1}
\end{array}\right]\left[\begin{array}{cc}
1 & \mathbf{0} \\
\mathbf{0} & \boldsymbol{W}_{2}
\end{array}\right]\left[\begin{array}{ccc}
\mathbf{0} & 1 & \mathbf{0} \\
\boldsymbol{I}_{L} & \mathbf{0} & \mathbf{0} \\
\mathbf{0} & \mathbf{0} & \boldsymbol{I}_{m-L-1}
\end{array}\right], \\
& \boldsymbol{V}_{3} \triangleq\left[\begin{array}{ll}
\boldsymbol{V}_{2} & 0 \\
0 & 1
\end{array}\right]\left[\begin{array}{cc}
\boldsymbol{I}_{L} & 0 \\
\boldsymbol{\alpha} & 1
\end{array}\right]
\end{aligned}
$$

Then $\boldsymbol{W}_{3}$ is an $m \times m$ permutation matrix and $\boldsymbol{V}_{3}$ is an $(L$ $+1) \times(L+1)$ nonsingular real matrix. From (D.4) $\sim(D .9)$, we obtain

$$
\begin{aligned}
\widetilde{\boldsymbol{B}}_{3} & \triangleq\left(\boldsymbol{V}_{3}\right)^{-1} \widetilde{\boldsymbol{B}}_{2} \boldsymbol{W}_{3}=\left(\boldsymbol{V}_{1} \boldsymbol{V}_{3}\right)^{-1} \widetilde{\boldsymbol{B}} \boldsymbol{W}_{1} \boldsymbol{W}_{3} \\
& =\left[\begin{array}{cccc}
\boldsymbol{I}_{L} & \mathbf{0} & -\boldsymbol{Y} & * \\
\mathbf{0} & 1 & \boldsymbol{\gamma} & *
\end{array}\right]
\end{aligned}
$$

where $\boldsymbol{\gamma} \triangleq\left[\gamma_{1}, \gamma_{2}, \cdots, \gamma_{t}\right]$ is a $1 \times t$ real matrix.

Hereafter we consider the following three cases.

Case $1: \gamma_{1}<0$

Let

$$
\begin{aligned}
\boldsymbol{W}_{4} \triangleq\left[\begin{array}{cccc}
\boldsymbol{I}_{L_{1}} & 0 & \mathbf{0} & \mathbf{0} \\
\mathbf{0} & \mathbf{0} & \boldsymbol{I}_{L-L_{1}} & \mathbf{0} \\
\mathbf{0} & 1 & \mathbf{0} & \mathbf{0} \\
\mathbf{0} & \mathbf{0} & \mathbf{0} & \boldsymbol{I}_{m-L-1}
\end{array}\right], \\
\boldsymbol{V}_{4} \triangleq\left[\begin{array}{ccc}
\boldsymbol{I}_{L_{1}} & 0 & \mathbf{0} \\
\mathbf{0} & \mathbf{0} & \boldsymbol{I}_{L-L_{1}} \\
\mathbf{0} & 1 & \mathbf{0}
\end{array}\right]
\end{aligned}
$$

Then $\boldsymbol{W}_{4}$ is an $m \times m$ permutation matrix and $\boldsymbol{V}_{4}$ is an $(L+1) \times(L+1)$ nonsingular real matrix.

From (D.7), (D.10), and (D.11), we obtain the following equations.

$$
\begin{aligned}
\widetilde{\boldsymbol{B}}_{4} & \triangleq\left(\boldsymbol{V}_{4}\right)^{-1} \widetilde{\boldsymbol{B}}_{3} \boldsymbol{W}_{4}=\left(\boldsymbol{V}_{1} \boldsymbol{V}_{3} \boldsymbol{V}_{4}\right)^{-1} \widetilde{\boldsymbol{B}} \boldsymbol{W}_{1} \boldsymbol{W}_{3} \boldsymbol{W}_{4} \\
& =\left[\boldsymbol{I}_{L+1}, \quad-\boldsymbol{Z}, *\right]
\end{aligned}
$$

where

$$
\begin{aligned}
& \boldsymbol{Z} \triangleq\left[\begin{array}{ccccc}
z_{1} & * & * & \cdots & * \\
\mathbf{0} & z_{2} & * & \cdots & * \\
& \cdots & \cdots & & \cdots \\
\mathbf{0} & \mathbf{0} & \cdots & \mathbf{0} & \boldsymbol{z}_{t}
\end{array}\right], \\
& 1 \leqq t \leqq M_{1}+M_{2}+\cdots+M_{t}=L+1, \\
& \boldsymbol{z}_{1} \triangleq\left[\boldsymbol{y}_{1}{ }^{T},-\gamma_{1}\right]^{T}>\mathbf{0}, M_{1} \triangleq L_{1}+1, \\
& \boldsymbol{z}_{i} \triangleq \boldsymbol{y}_{i}>\mathbf{0}, M_{i} \triangleq L_{i} ; i=2,3, \cdots, t
\end{aligned}
$$

It is evident that $\boldsymbol{W}_{1} \boldsymbol{W}_{3} \boldsymbol{W}_{4}$ is an $m \times m$ permutation matrix and $\boldsymbol{V}_{1} \boldsymbol{V}_{3} \boldsymbol{V}_{4}$ is an $(L+1) \times(L+1)$ nonsingular real matrix. This means that necessity is valid when $F=L+1$ in Case 1.

Case $2: \gamma_{1}=0$

In this case, (D.10) can be written as

$$
\widetilde{\boldsymbol{B}}_{3}=\left[\begin{array}{cccc}
\boldsymbol{I}_{L_{1}} & \mathbf{0} & -\boldsymbol{y}_{1} & \boldsymbol{y}_{12} \\
\mathbf{0} & \boldsymbol{I}_{L-L_{1}+1} & \mathbf{0} & \boldsymbol{G}_{3}
\end{array}\right]
$$

where 


$$
\begin{aligned}
\boldsymbol{G}_{3} \triangleq\left[\begin{array}{ccccc}
-\boldsymbol{y}_{2} & * & \cdots & * & * \\
\mathbf{0} & -\boldsymbol{y}_{3} & \cdots & * & * \\
\cdots & \cdots & & \cdots & \\
\mathbf{0} & \mathbf{0} & \cdots & -\boldsymbol{y}_{t} & * \\
\gamma_{2} & \gamma_{3} & \cdots & \gamma_{t} & *
\end{array}\right], \\
\boldsymbol{y}_{12} \in \boldsymbol{R}^{L_{1 \times \cdot m-L-2)}}, \quad \boldsymbol{G}_{3} \in \boldsymbol{R}^{\left(L-L_{1}+1\right) \times(m-L-2)}
\end{aligned}
$$

Next let

$$
\boldsymbol{W}_{5} \triangleq\left[\begin{array}{ccc}
\boldsymbol{I}_{L+1} & \mathbf{0} & \mathbf{0} \\
\mathbf{0} & \mathbf{0} & 1 \\
\mathbf{0} & \boldsymbol{I}_{m-L-2} & \mathbf{0}
\end{array}\right]
$$

Then from (D.10) and (D.14) (D.16), we have

$$
\widetilde{\boldsymbol{B}}_{5} \triangleq \widetilde{\boldsymbol{B}}_{3} \boldsymbol{W}_{5}=\left(\boldsymbol{V}_{1} \boldsymbol{V}_{3}\right)^{-1} \widetilde{\boldsymbol{B}} \boldsymbol{W}_{1} \boldsymbol{W}_{2} \boldsymbol{W}_{5}
$$

$$
=\left[\begin{array}{ccc}
\boldsymbol{I}_{L_{1}} & \mathbf{0} & \boldsymbol{G}_{4} \\
\mathbf{0} & \boldsymbol{I}_{L-L_{1}+1} & \boldsymbol{G}_{5}
\end{array}\right]
$$

where

$$
\begin{aligned}
& \boldsymbol{G}_{4} \triangleq\left[\boldsymbol{y}_{12}, \quad-\boldsymbol{y}_{1}\right] \in \boldsymbol{R}^{L_{1} \times(m-L-1)}, \\
& \boldsymbol{G}_{5} \triangleq\left[\boldsymbol{G}_{3}, \quad \mathbf{0}\right] \in \boldsymbol{R}^{\left(L-L_{1}-1\right) \times(m-L-1)}
\end{aligned}
$$

Since system $\widetilde{\boldsymbol{B}}$ is $p$. $c$., system $\widetilde{\boldsymbol{B}}_{5}$ is $p$. c . from Theorem 4. Furthermore system $\boldsymbol{B}^{*}{ }_{2}$ is $p$. c. from Lemma 5 , where

$$
\boldsymbol{B}^{\#}{ }_{2} \triangleq\left[\boldsymbol{I}_{L-L_{1}+1}, \quad \boldsymbol{G}_{5}\right] \in \boldsymbol{R}^{\left(L-L_{1}-1\right) \times\left(m-L_{1}\right)}
$$

If wet

$$
\boldsymbol{B}^{\#}{ }_{3} \triangleq\left[\boldsymbol{I}_{L-L_{1}+1}, \quad \boldsymbol{G}_{3}\right] \in \boldsymbol{R}^{\left(L-L_{1}-1\right) \times\left(m-L_{1}-1\right)}
$$

then noting (D.18) and Lemma 4 , system $\boldsymbol{B}^{*}{ }_{3}$ is p. c.. Since $L-L_{1}+1 \leqq L$, we can apply the hypothesis to system $\boldsymbol{B}_{3}{ }_{3}$. Thus there exist positive integers $\left\{r, M_{2}, M_{3}, \cdots, M_{r}\right\}$, an $\left(m-L_{1}-1\right) \times\left(m-L_{1}-1\right)$ permutation matrix $\boldsymbol{W}_{6}$, an $(L$ $\left.-L_{1}+1\right) \times\left(L-L_{1}+1\right)$ nonsingular real matrix $V_{6}$, and $M_{i}$ $\times 1$ positive vectors $z_{i} ;(i=2,3, \cdots, r)$ such that

$$
\left(\boldsymbol{V}_{6}\right)^{-1} \boldsymbol{B}^{*}{ }_{3} \boldsymbol{W}_{6}=\left[\boldsymbol{I}_{L-L_{1}+1},-\boldsymbol{Z}_{0}, *\right]
$$

where $\boldsymbol{Z}_{0}$ is an $\left(L-L_{1}+1\right) \times(r-1)$ real matrix defined as

$$
Z_{0} \triangleq\left[\begin{array}{ccccc}
z_{2} & * & * & \cdots & * \\
0 & z_{3} & * & \cdots & * \\
& \cdots & \cdots & & \cdots \\
0 & 0 & \cdots & 0 & z_{r}
\end{array}\right]
$$

$$
1 \leqq r-1 \leqq M_{2}+M_{3}+\cdots+M_{r}=L-L_{1}+1
$$

with $*$ denoting some real matrix of appropriate size.

Next, let

$\left[0, \boldsymbol{y}_{12}\right] \boldsymbol{W}_{6} \triangleq[\xi, *], \quad \xi \in \boldsymbol{R}^{L_{1} \times\left(L-L_{1}-1\right)}$,

$$
\begin{aligned}
& \boldsymbol{V}_{7} \triangleq\left[\begin{array}{cc}
\boldsymbol{I}_{L_{1}} & \mathbf{0} \\
\mathbf{0} & \boldsymbol{V}_{6}
\end{array}\right]\left[\begin{array}{ccc}
\boldsymbol{I}_{L_{1}} & \boldsymbol{\xi} \\
\mathbf{0} & \boldsymbol{I}_{L-L_{1}+1}
\end{array}\right], \\
& \boldsymbol{W}_{7} \triangleq\left[\begin{array}{ccc}
\boldsymbol{I}_{L_{1}} & \mathbf{0} & \mathbf{0} \\
\mathbf{0} & \boldsymbol{W}_{6} & \mathbf{0} \\
\mathbf{0} & \mathbf{0} & 1
\end{array}\right]\left[\begin{array}{ccc}
\boldsymbol{I}_{L+1} & \mathbf{0} & \mathbf{0} \\
\mathbf{0} & \mathbf{0} & \boldsymbol{I}_{M-L-2} \\
\mathbf{0} & 1 & \mathbf{0}
\end{array}\right]
\end{aligned}
$$

Then from (D.17) (D.24), we have the following equation.

$$
\begin{aligned}
\widetilde{\boldsymbol{B}}_{7} & \triangleq\left(\boldsymbol{V}_{7}\right)^{-1} \widetilde{\boldsymbol{B}}_{5} \boldsymbol{W}_{7}=\left(\boldsymbol{V}_{1} \boldsymbol{V}_{3} \boldsymbol{V}_{7}\right)^{-1} \tilde{\boldsymbol{B}} \boldsymbol{W}_{1} \boldsymbol{W}_{3} \boldsymbol{W}_{5} \boldsymbol{W}_{7} \\
& =\left[\begin{array}{lll}
\boldsymbol{I}_{L+1}, & -\boldsymbol{Z}, \quad *
\end{array}\right]
\end{aligned}
$$

$$
\begin{aligned}
& \boldsymbol{Z} \triangleq\left[\begin{array}{ccccc}
\boldsymbol{z}_{1} & * & * & \cdots & * \\
\mathbf{0} & \boldsymbol{z}_{2} & * & \cdots & * \\
& \cdots & \cdots & & \cdots \\
\mathbf{0} & \mathbf{0} & \cdots & \mathbf{0} & \boldsymbol{z}_{r}
\end{array}\right] \\
& 1 \leqq r \leqq M_{1}+M_{2}+\cdots+M_{r}=L+1, \\
& \boldsymbol{z}_{1} \triangleq \boldsymbol{y}_{1}>\mathbf{0}, \quad M_{1} \triangleq L_{1}, \\
& \boldsymbol{z}_{i}>\mathbf{0} ; i=2,3, \cdots, r
\end{aligned}
$$

Since $\boldsymbol{W}_{1} \boldsymbol{W}_{3} \boldsymbol{W}_{5} \boldsymbol{W}_{7}$ is an $m \times m$ permutation matrix and $\boldsymbol{V}_{1} \boldsymbol{V}_{3} \boldsymbol{V}_{7}$ is an $(L+1) \times(L+1)$ nonsingular real matrix, the necessity is valid when $F=L+1$ in Case 2 .

Case $3: \gamma_{1}>0$

Let

$$
\begin{aligned}
& \boldsymbol{V}_{\mathbf{8}} \triangleq\left[\begin{array}{ccc}
\boldsymbol{I}_{L_{1}} & \mathbf{0} & -\boldsymbol{y}_{1} \\
\mathbf{0} & \boldsymbol{I}_{L-L_{1}} & \mathbf{0} \\
\mathbf{0} & \mathbf{0} & \gamma_{1}
\end{array}\right] \\
& \boldsymbol{W}_{8} \triangleq\left[\begin{array}{ccc}
\boldsymbol{I}_{L} & \mathbf{0} & \mathbf{0} \\
\mathbf{0} & \mathbf{0} & 1 \\
\mathbf{0} & \boldsymbol{I}_{m-L-1} & \mathbf{0}
\end{array}\right]
\end{aligned}
$$

Then from (D.10) and (D.27) we obtain

$$
\widetilde{\boldsymbol{B}}_{8} \triangleq\left(\boldsymbol{V}_{8}\right)^{-1} \widetilde{\boldsymbol{B}}_{3} \boldsymbol{W}_{8}=\left(\boldsymbol{V}_{1} \boldsymbol{V}_{3} \boldsymbol{V}_{8}\right)^{-1} \widetilde{\boldsymbol{B}} \boldsymbol{W}_{1} \boldsymbol{W}_{3} \boldsymbol{W}_{8}
$$$$
\doteq\left[\widetilde{\boldsymbol{B}}_{9}, \boldsymbol{y}^{*}\right]
$$

where
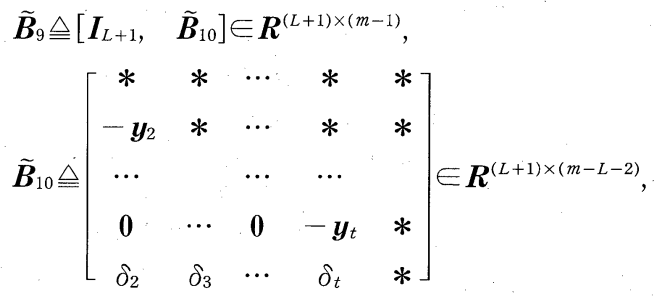

$\delta \triangleq\left(\gamma_{1}\right)^{-1}>0$,

$\boldsymbol{y}^{*} \triangleq\left[\left(\boldsymbol{y}_{1}^{*}\right)^{T}, \quad \mathbf{0}, \quad \delta\right]^{T} \in \boldsymbol{R}^{(L+1) \times 1}$,

$\delta_{i} \triangleq \delta \gamma_{i} ; i=2,3, \cdots, t$;

$\boldsymbol{y}_{1}{ }^{*} \triangleq \delta \boldsymbol{y}_{1}>0$

Since system $\widetilde{\boldsymbol{B}}$ is $p$. c., system $\widetilde{\boldsymbol{B}}_{8}$ is $p$. c. from Theorem 4. On the other hand $\boldsymbol{y}^{*}$ in (D.28) is nonnegative from (D.29). Thus system $\widetilde{\boldsymbol{B}}_{9}$ is $p$. c. by Lemma 4. Therefore in this Case 3 , the positive controllability of $m$ input system reduces to that of $(m-1)$ input system. If the same procedures are repeated, only Case 3 will survive. But, the number of $m$ reduces by one, for each occurrence of Case 3. Therefore, the necessity may be reduced ultimately to the case of $m=F+1$, which is valid by Lemma 3 . Hence the necessity is valid when $F=L+1$ in Case 3. Q.E.D.

where 


\section{Hiroshi Yoshida (Member)}

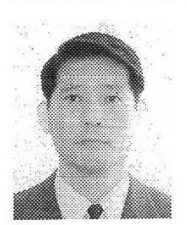

He received the Dr. Eng. degree in electronic engineering from Kyushu University, Fukuoka, Japan, in 1979. During 1966 and 1979, he was a research associate with the Department of Electronic Engineering, Kyushu University. From 1980 to 1987, he was an assistant professor with the Department of Electronic Engineering, Kagoshima University. Since 1988, he has been a professor at Kagoshima University. His main field of interest lies on sampled-data control system theory.

$\mathrm{He}$ is a member of IEEE, Institute of Electronics, Information and Communication Engineers of Japan and Society of Instrument and Control Engineers of Japan.

\section{Tetsuro Tanaka (Member)}

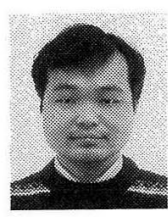

He was born in Kagoshima, Japan in 1961. He received the B. S., M. S., and Dr. Eng. degrees in electronic engineering from Kyushu University, Fukuoka, Japan, in 1983, 1985 and 1992 respectively. He is an assistant professor at Kagoshima University. His current interest is in the area of switching power-converter, especially, noise reduction with random process.

He is a member of IEEE, Institute of Elec tronics, Information and Communication Engineers of Japan and Society of Instrument and Control Engineers of Japan.

\section{Kazutomo YUNOKUCHI}

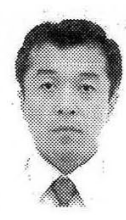

In 1987 he stayed at Institute of Medical Engineering, University of Tokyo. He worked at Francis Bitter Magnet Laboratory in M. I. T. as a visiting researcher from 1989 to 1991. He received Dr. Engineering degree from University of Tokyo in 1993. He is a professor of Department of Bioengineering, Kagoshima University. His research interest concentrates on the control system of human body.

$\mathrm{He}$ is a member of IEEE, Institute of Electronics, Information and Communication Engineers of Japan and Institute of Electrical Enginecrs of Japan and the Japan society of medical engineering. 